\title{
Phytoprotection
}

\section{Bioécologie de Megarhyssa atrata [Hymenoptera : Ichneumonidae], ectoparasite de Tremex columba [Hymenoptera : Siricidae] au Québec}

\section{J.-P. Nénon}

Volume 76, numéro 3, 1995

URI : https://id.erudit.org/iderudit/706090ar

DOI : https://doi.org/10.7202/706090ar

Aller au sommaire du numéro

Éditeur(s)

Société de protection des plantes du Québec (SPPQ)l

ISSN

0031-9511 (imprimé)

1710-1603 (numérique)

Découvrir la revue

Citer cet article

Nénon, J.-P. (1995). Bioécologie de Megarhyssa atrata [Hymenoptera :

Ichneumonidae], ectoparasite de Tremex columba [Hymenoptera : Siricidae] au

Québec. Phytoprotection, 76(3), 115-122. https://doi.org/10.7202/706090ar
Résumé de l'article

Dans le sud du Québec, Megarhyssa atrata vole de mai à septembre avec un pic nettement marqué durant la première quinzaine de juin; les mâles

apparaissent dès le 19 mai, les femelles sont rencontrées jusqu'au 9 septembre. Durant l'été 1992, $M$. atrata a représenté 50\% du peuplement constitué par trois espèces de Megarhyssa sympatriques et inféodées au même hôte, Tremex columba, en développement dans l'orme américain (Ulmus americana). Par contre, $M$. atrata représentait $60 \%$ de la population totale de femelles des trois espèces. Le taux de parasitisme estimé sur 107 individus-hôtes observés était de 57,9 \%. M. atrata se développe en ectoparasitoïde et son cycle, qui est probablement de 2 ans, comprend quatre stades larvaires. M. atrata apparaît comme un élément de régulation des populations de Tremex columba et pourrait être un agent de lutte biologique en foresterie. Ces données sur l'écobiologie de M. atrata sont les premières pour le Canada. 


\title{
Bioécologie de Megarhyssa atrata [Hymenoptera : Ichneumonidae], ectoparasitoïde de Tremex columba [Hymenoptera : Siricidae] au Québec
}

\author{
Jean-Pierre Nénon ${ }^{1}$
}

Reçu 1994-12-02; accepté 1996-03-15

Dans le sud du Québec, Megarhyssa atrata vole de mai à septembre avec un pic nettement marqué durant la première quinzaine de juin; les mâles apparaissent dès le 19 mai, les femelles sont rencontrées jusqu'au 9 septembre. Durant l'été $1992, M$. atrata a représenté $50 \%$ du peuplement constitué par trois espèces de Megarhyssa sympatriques et inféodées au même hôte, Tremex columba, en développement dans l'orme américain (UImus americana). Par contre, $M$. atrata représentait $60 \%$ de la population totale de femelles des trois espèces. Le taux de parasitisme estimé sur 107 individus-hôtes observés était de $57,9 \%$. M. atrata se développe en ectoparasitoïde et son cycle, qui est probablement de 2 ans, comprend quatre stades larvaires. $M$. atrata apparaît comme un élément de régulation des populations de Tremex columba et pourrait être un agent de lutte biologique en foresterie. Ces données sur l'écobiologie de $M$. atrata sont les premières pour le Canada.

Nénon J.-P. 1995. Bioecology of Megarhyssa atrata [Hymenoptera : Ichneumonidae], an ectoparasitoid of Tremex columba [Hymenoptera : Siricidae] in Quebec. PHYTOPROTECTION 76 : 115-122.

In southern Quebec, Megarhyssa atrata flies from May to September with a marked peak during the first 2 wk of June; males appear as soon as 19 May, and females are found as late as 9 September. During the summer 1992, $M$. atrata represented $50 \%$ of the population composed of three sympatric species of Megarhyssa sharing a common host, Tremex columba, developing in the American elm (UImus americana). However, M. atrata represented $60 \%$ of the total female population of the three species. The rate of parasitism estimated on the 107 host individuals examined was $57.9 \%$. M. atrata develops as an ectoparasitoid and his life cycle, that probably lasts $2 \mathrm{yr}$, has four larval stages. M. atrata acts as a regulatory factor for Tremex columba populations, and could be used as a biological control agent in forestry. These data on the ecobiology of $M$. atrata are the first to be reported for Canada.

\section{INTRODUCTION}

Malgré leur vaste répartition boréale néarctique et holarctique et leur grande taille, les Megarhyssa spp. [Hymenoptera: Ichneumonidae] restent des Hyménoptères largement méconnus ne faisant l'objet que de données relative- ment peu nombreuses collectées depuis la fin du $19^{\mathrm{e}}$ siècle (Harrington 1887; Riley 1888). Elles concernent la systématique du genre (Townes et Townes 1960), celle des hôtes (Aubert 1969; Cameron 1965) et surtout le comportement spectaculaire d'accouplement et de ponte (Abbott 1934, 1936; Barlow 1921; Fattig 1949; Fyles 1916;

1. Université de Rennes 1, Laboratoire d'écobiologie des insectes parasitoïdes, Campus de Beaulieu, 35042 Rennes Cédex, France 
Harrington 1887; Marchand 1921; Riley 1888). Les données plus récentes (Heatwole et Davis 1965; Heatwole et al. 1962, 1965) fournissent des informations sur l'écologie de ces insectes au Michigan, reprises par Gibbons (1979) pour discuter le principe d'exclusion compétitive établi par Hardin (1960).

Nos travaux visent à progresser dans la connaissance de la biologie et de l'écologie de Megarhyssa atrata F. [Hymenoptera : Ichneumonidae], évaluer son importance dans le peuplement nordappalachien constitué par trois espèces sympatriques aisément identifiables grâce à une nouvelle clé de détermination (Nénon 1993), connaître les modalités de son parasitisme et son impact parasitaire sur les hôtes Siricidae. Nous avons mené des prospections de terrain au Québec au sud de Montréal durant l'été 1992 et exploité les données fournies par trois collections entomologiques du Québec et de l'Ontario.

\section{MATÉRIEL ET MÉTHODES}

\section{Période de capture de $M$. atrata}

Pour connaître la période de vol de I'espèce, I'inventaire des collections d'Agriculture et Agroalimentaire Canada à Saint-Jean-sur-Richelieu (Québec) et Ottawa (Ontario) et de celle du musée Lyman du campus Macdonald de I'Université McGill à Sainte-Anne-de-Bellevue (Québec) a été effectué; il permet de connaître les dates de capture de 94 échantillons provenant du sud-est du Québec. Nos observations de terrain concernent 60 individus et ont été faites à Mont-Saint-Hilaire (lat. $45^{\circ} 33^{\prime} \mathrm{N}$ long. $73^{\circ} 10^{\prime}$ W), à Sainte-Anne-de-Bellevue (lat. $45^{\circ} 25^{\prime} \mathrm{N}$ long. $75^{\circ} 56^{\prime} \mathrm{W}$ ) et surtout à Sherrington (lat. $45^{\circ} 10^{\prime} \mathrm{N}$ long. $73^{\circ} 31^{\prime} \mathrm{W}$ ).

\section{Importance relative de $M$. atrata dans le peuplement}

Du 5 au 25 juin 1992, nous avons prospecté deux parcelles boisées d'une superficie totale d'environ 40 ha situées sur le territoire de Sherrington en parcourant systématiquement le même itinéraire, selon les conditions météorologiques, de 1 à 5 fois par jour, entre 08:00 et 20:00. Chacun de ces échantil-
Ionnages a permis d'observer 30 ormes (Ulmus americana L.) de grande taille. Vingt-sept parcours de comptage ont été ainsi effectués, chacun en $1 \mathrm{~h} 30 \mathrm{~min}$. Tous les $M$. atrata au vol ou posés ont été comptés en distinguant les sexes.

\section{Taux de parasitisme de l'hôte Tremex columba}

Tremex columba L. [Hymenoptera :Siricidae] est un Hyménoptère symphyte dont les larves xylophages se rencontrent fréquemment dans les ormes. L'un des ormes s'étant révélé particulièrement attractif pour le $M$. atrata a été abattu le 25 juin 1992 au soir; la partie basse du tronc a été débitée en rondelles de 18 à $20 \mathrm{~cm}$. Le diamètre de l'arbre était de $0,4 \mathrm{~m}$ à $0,4 \mathrm{~m}$ de hauteur. Le trait de sciage par la tronçonneuse avait une épaisseur de $2 \mathrm{~cm}$. Les deux tronçons les plus bas ont été conservés comme témoins pour suivre d'éventuelles émergences. Les neuf autres, remontant jusqu'à une hauteur de 2,50 m, correspondant à la zone activement prospectée par $M$. atrata ont été éclatés du 26 au 29 juin au ciseau à bois afin de recueillir les T. columba et les $M$. atrata présents, en notant l'état de développement de l'hôte et des parasitoïdes associés. Le volume de bois des neuf tronçons examinés est de $0,263 \mathrm{~m}^{3}$.

\section{Cycle de développement du M. atrata}

Il est actuellement impossible de distinguer les larves des différentes espèces. Nous n'avons donc pris en considération que les larves ou nymphes trouvées à plus de $10 \mathrm{~cm}$ de profondeur dans le bois, afin d'éliminer les larves des espèces à ovipositeur plus court que celui de $M$. atrata. Les $T$. columba sains ou parasités, les larves en fin de cycle et les nymphes de $M$. atrata trouvées seules dans la galerie de l'hôte ont été conservées dans I'alcool (70 \% vol). UItérieurement, les individus des différents stades ont été séchés sur papier filtre et pesés sur une balance Sartorius type $\mathrm{L}$ 220 (précision de $\pm 1 \mathrm{mg}$ ); leur longueur a été mesurée sur papier millimétrique. Les hypothèses sur la durée du cycle de développement sont en partie fondées sur des observations de terrain sommairement effectuées en juillet 1991 sur le site échantillonné en 1992. 


\section{RÉSULTATS ET DISCUSSION}

\section{Période de capture des $M$. atrata mâles et femelles}

L'inventaire des trois collections a permis de recenser 94 individus; ils ont été collectés durant une période de 80 ans (1912-1992). M. atrata est capturé du 12 mai en 1941 au 9 septembre en 1935 dans la zone sud du Québec. Des dates plus précoces de vol sont obtenues plus au sud, aux États-Unis : 9 mai 1920 en Pennsylvanie (Champlain 1921) et 30 avril 1948 en Georgie (Fattig 1949). Comme nos observations concernent 60 individus, 154 individus au total sont donc pris en considération.

Dans la région étudiée, $90,9 \%$ de la population est observée entre le $1^{\text {er }}$ juin et le 10 juillet, et $81,7 \%$ durant le seul mois de juin (fig. 1). Les histogrammes montrent une précocité de la présence des mâles par rapport aux femelles, beaucoup plus nombreuses en juillet alors que les mâles se raréfient. La capture des mâles cesse au début de juillet.

De telles données sont très fortement influencées par les conditions climatiques, le vent et la pluie s'opposant au vol des insectes; elles peuvent donc varier fort sensiblement d'une année à l'autre. La période de vol et toute l'activité en général des adultes est fortement liée aux conditions ambiantes. Nos observations confirment l'existence de deux pics journaliers d'activité de ponte entre 10:00 et 12:00, ainsi qu'entre 16:00 et 17:00, déjà signalés par Heatwole et Davis (1965) qui notaient par ailleurs une nette relation entre la conjonction hygrométrietempérature et l'activité de l'espèce. Ces pics quotidiens d'activité n'ont toutefois lieu qu'en absence de vent fort mais dont la vitesse n'a pas été mesurée. Aucun individu n'a été observé en vol par une température inférieure à $14^{\circ} \mathrm{C}$ et la ponte $\mathrm{n}^{\prime}$ a pas été observée au-dessous de $16^{\circ} \mathrm{C}$. Les regroupements de mâles aux sites d'émergence des femelles et les accouplements ont été observés le matin.

\section{Importance relative de $M$. atrata dans le peuplement des Megarhyssa} Durant le mois de juin 1992, 116 individus appartenant à trois espèces du genre Megarhyssa ont été observés sur une surface d'environ 40 ha (tableau 1). Les deux sexes étant confondus, $M$. atrata constituait $50 \%$ du peuplement, Megarhyssa macrurus L., $41,4 \%$ et Megarhyssa greeni Viereck, 8,6\%. Megarhyssa nortoni Cresson considéré comme rare par Fyles (1916) n'a pas été observé. Au sein de l'ensemble des femelles, celles de $M$. atrata représentent $60,3 \%$, celles de $M$. macrurus, $30,2 \%$ et celles de $M$. greeni, $9,5 \%$.

Les variations d'importance relative entre les espèces constatées durant le mois de juin 1992 sur la seule localité de Sherrington diffèrent sensiblement de celles publiées antérieurement pour la région du sud de Montréal et pour une période de 80 ans (Nénon 1993). Elles s'expliquent au moins par deux raisons; le nombre des échantillons considérés reste relativement faible et par ailleurs, les observations menées en 1992 sur la parcelle de Sherrington reflètent une situation momentanée que l'on sait évolutive dans le temps. Heatwole et Davis (1965) mettent en évidence au Michigan, trois états de structure du peuplement de Megahryssa marqués par la prédominance d'une ou l'autre espèce en liaison avec l'état de décomposition des arbres morts qui abritent l'hôte, T. columba. Dans cette optique, notre parcelle d'étude en 1992 s'inscrit dans le stade 2 ou 3 de Heatwole et Davis où $M$. atrata domine dans le peuplement.

\section{Parasitisme de l'hôte : taux et modalités}

L'éclatement de $0,263 \mathrm{~m}^{3}$ de bois d'orme a permis d'observer 107 situations se répartissant ainsi : 45 larves de différents stades de $T$. columba non parasitées, 34 larves de $T$. columba parasitées par une jeune larve de $M$. atrata , 23 larves âgées et 5 prénymphes ou nymphes de $M$. atrata. Les larves âgées, les prénymphes et nymphes ont été trouvées seules dans la galerie du $T$. columba qui est entièrement consommée ou ne subsiste que par des vestiges de cuticule. Le tableau 2 montre la répartition verticale des individus de Megarhyssa spp. trouvés dans les neuf tronçons constituant la partie basse d'un orme. Les deux tronçons témoins de la partie la plus basse du tronc, conservés pour contrôler d'éventuelles émergences de M. atrata en 1993 n'ont pu être 

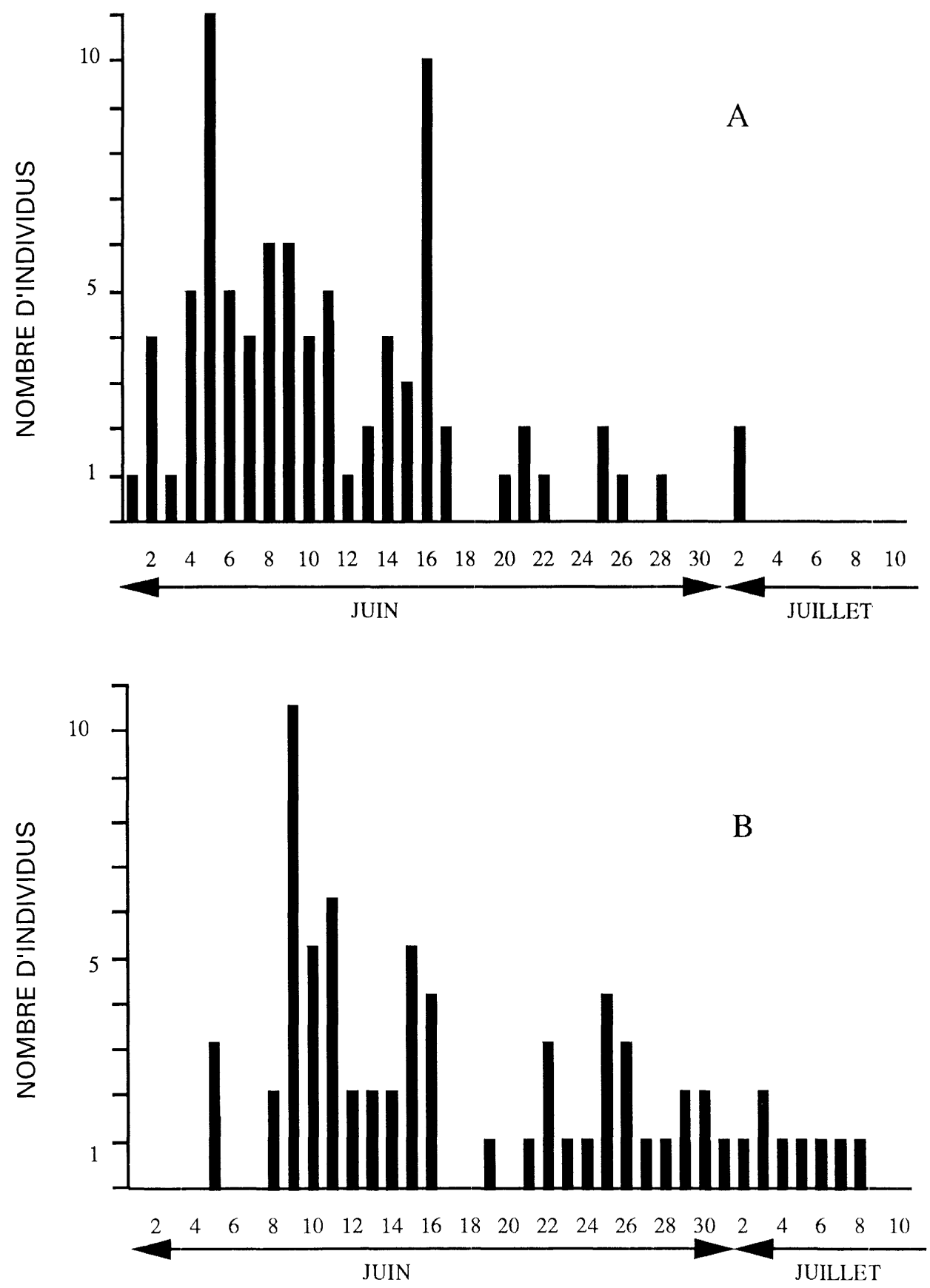

Figure 1. Répartition des captures quotidiennes de $M$. atrata mâles (A) et femelles (B) durant les mois de juin et de juillet (période 1912-1992) d'après la somme des individus trouvés dans les collections entomologiques et de ceux de nos propres observations. 
Tableau 1. Structure du peuplement constitué par les adultes de trois espèces de Megarhyssa à Sherrington (Québec) en juin 1992

\begin{tabular}{|c|c|c|c|c|c|c|c|c|}
\hline \multirow[b]{2}{*}{ Date } & \multirow{2}{*}{$\begin{array}{c}N^{b r e} \\
\text { d'échantillon- } \\
\text { nages }\end{array}$} & \multicolumn{2}{|c|}{ M. atrata } & \multicolumn{2}{|c|}{ M. macrurus } & \multicolumn{2}{|c|}{ M. greeni } & \multirow[b]{2}{*}{$\begin{array}{l}\mathrm{N}^{\text {bre }} \text { total d'individus } \\
\text { observés }\end{array}$} \\
\hline & & $\begin{array}{c}\mathrm{n}^{\text {bre }} \text { de } \\
\text { femelles }\end{array}$ & $\begin{array}{l}\mathrm{n}^{\text {bre }} \mathrm{de} \\
\text { mâles }\end{array}$ & $\begin{array}{c}n^{\text {bre }} \text { de } \\
\text { femelles }\end{array}$ & $\begin{array}{l}\mathrm{n}^{\text {bre }} \mathrm{de} \\
\text { mâles }\end{array}$ & $\begin{array}{c}\mathrm{n}^{\text {bre }} \text { de } \\
\text { femelles }\end{array}$ & $\begin{array}{l}\mathrm{n}^{\text {bre }} \mathrm{de} \\
\text { mâles }\end{array}$ & \\
\hline $1992-06-05$ & 5 & 3 & 10 & 0 & 0 & 1 & 1 & 15 \\
\hline $1992-06-08$ & 1 & 0 & 0 & 2 & 9 & 0 & 0 & 11 \\
\hline $1992-06-09$ & 3 & 9 & 0 & 1 & 0 & 0 & 0 & 10 \\
\hline $1992-06-10$ & 3 & 0 & 0 & 0 & 0 & 0 & 1 & 1 \\
\hline $1992-06-11$ & 3 & 6 & 4 & 0 & 0 & 0 & 0 & 10 \\
\hline $1992-06-12$ & 2 & 3 & 0 & 7 & 13 & 0 & 0 & 23 \\
\hline $1992-06-15$ & 2 & 6 & 1 & 2 & 0 & 0 & 0 & 9 \\
\hline $1992-06-16$ & 3 & 8 & 3 & 2 & 0 & 3 & 2 & 18 \\
\hline $1992-06-23$ & 2 & 0 & 0 & 5 & 6 & 1 & 0 & 12 \\
\hline $1992-06-25$ & 3 & 3 & 2 & 0 & 1 & 1 & 0 & 7 \\
\hline Total & 27 & 38 & 20 & 19 & 29 & 6 & 4 & 116 \\
\hline
\end{tabular}

suivis avec précision; ils n'auraient fourni aucun adulte.

En admettant que chaque larve âgée ou prénymphe ou nymphe a consommé une larve de $T$. columba, le taux de parasitisme de $M$. atrata est de $57,9 \%$ (tableau 2). Cette donnée est la deuxième pour le continent américain après celle de Heatwole et Davis (1965) qui rapportent au Michigan un taux de 26,7 \% pour 86 larves de T. columba observées.

Les $M$. atrata sont toujours trouvés en situation d'ectoparasitisme, attachés ou détachés de l'hôte, ce qui est conforme aux données de Cushman (1926). Cet ectoparasitoïde est solitaire; aucune trace d'une éventuelle compétition intraspécifique n'a été observée; il n'existe actuellement pas de données sur le nombre d'oeufs pondus à chaque insertion de l'ovipositeur.

Alors qu'aucune régulation biotique ne semble s'exercer sur la population larvaire de $M$. atrata, il est probable que cette régulation est très importante sur les femelles adultes. De nombreux ovipositeurs restent fichés dans les troncs après prédation. La séquence forage par l'ovipositeur - ponte - retrait de l'ovipositeur constitue en effet une longue phase d'immobilisation de la femelle, alors exposée à la prédation active par les oiseaux. Cette prédation a été ob- servée mais non quantifiée. Elle se produit tout particulièrement lorsque se déploient les membranes intersegmentaires abdominales, jaunes et translucides, constituant le système d'entraînement du stylet de l'ovipositeur (Nénon et al., données non publiées). Drooz (1985) signale que des femelles meurent parfois, $n$ 'ayant pas réussi à dégager leur ovipositeur du bois. Cette forme de régulation de la population est tout à fait originale au sein de la grande diversité des Hyménoptères parasitoïdes.

\section{Cycle de développement}

Actuellement, rien ne permet de distinguer les larves des différentes espèces de Megarhyssa. Nous avons sélectionné dans nos données 36 cas, attribués à $M$. atrata, en liaison avec la longueur de son ovipositeur; ces individus ont été obtenus à plus de $10 \mathrm{~cm}$ de la surface du tronc donc a priori, à une profondeur non accessible aux autres espèces car leur ovipositeur est trop court. En reportant sur un graphique orthonormé, le poids et la longueur de chaque larve, nous avons mis en évidence l'existence de quatre stades larvaires précédant le stade prénymphal, puis nymphal (fig. 2).

Nos observations laissent supposer que le cycle des $M$. atrata s'effectue sur 2 années; au cours de la première année, se dérouleraient les quatre stades larvaires (juin à juin) puis surviendrait la 
Tableau 2. Répartition verticale dans un tronc d'orme des larves de $T$. columba saines et parasitées par $M$. atrata (26 juin 1992)

\begin{tabular}{|c|c|c|c|c|c|c|c|}
\hline \multirow{2}{*}{$\begin{array}{l}\text { Hauteur } \\
\text { du tronçon } \\
\text { d'orme par } \\
\text { rapport au sol } \\
\text { (cm) }\end{array}$} & \multirow{2}{*}{$\begin{array}{c}\text { Tremex columba } \\
n^{\text {bre }} \text { de larves } \\
\text { non parasitées }\end{array}$} & \multicolumn{4}{|c|}{ Megarhyssa atrata } & \multicolumn{2}{|c|}{$\begin{array}{c}\text { Taux de parasitisme } \\
\text { de } T \text {. columba }\end{array}$} \\
\hline & & $\begin{array}{c}\mathrm{n}^{\text {bre }} \text { de } \\
\text { jeunes larves }\end{array}$ & $\begin{array}{c}\mathrm{n}^{\text {bre }} \text { de } \\
\text { larves âgées }\end{array}$ & $\begin{array}{c}n^{\text {bre }} \text { de } \\
\text { nymphes }\end{array}$ & $\begin{array}{c}\mathrm{n}^{\text {ore }} \text { total } \\
\mathrm{d}^{\text {'individus }}\end{array}$ & $\mathrm{M} / \mathrm{T}^{\mathrm{a}}$ & $\%$ \\
\hline $200-220$ & 1 & 0 & 0 & 0 & 0 & & 0 \\
\hline $180-200$ & 2 & 3 & 2 & 1 & 6 & $6 / 8$ & 75 \\
\hline $160-180$ & 3 & 3 & 3 & 0 & 6 & $6 / 9$ & 66 \\
\hline $140-160$ & 3 & 9 & 5 & 0 & 14 & $14 / 17$ & 82 \\
\hline $120-140$ & 2 & 5 & 1 & 1 & 7 & $7 / 9$ & 77 \\
\hline $100-120$ & 10 & 5 & 3 & 2 & 10 & $10 / 20$ & 50 \\
\hline $80-100$ & 5 & 7 & 9 & 0 & 16 & $16 / 21$ & 76 \\
\hline $60-80$ & 17 & 2 & 0 & 1 & 3 & $3 / 20$ & 15 \\
\hline $40-60$ & 2 & 0 & 0 & 0 & 0 & $0 / 2$ & 0 \\
\hline Total & 45 & 34 & 23 & 5 & 62 & $62 / 107$ & 57,9 \\
\hline
\end{tabular}

a $\mathrm{M} / \mathrm{T}=$ Rapport du nombre d'individus de $M$. atrata sur le nombre total d'individus de $T$. columba parasités et non parasités.

nymphose (fin juin - début juillet) et l'émergence l'année suivante. Ainsi pourrait être expliquée la coexistence, fin juin, d'adultes volants et, dans l'arbre examiné, de larves de tous stades de 5 à $35 \mathrm{~mm}$, d'une prénymphe et de cinq nymphes non pigmentées de $M$. atrata. Selon Baker (1972), Tremex columba est un insecte univoltin aux États-Unis et seIon Stilwell (1966), bivoltin au NouveauBrunswick (Canada). Nos observations laissent penser que le cycle de l'hôte semble se dérouler au Québec sur 2 années consécutives. Plusieurs femelles ont en effet été capturées en juillet 1991 au piège Malaise autour d'un orme qui n'était plus fréquenté par les $M$. atrata mais qui a donné lieu à plusieurs émergences du parasitoïde en 1992. En hypothèse, il est probable que les $M$. atrata infesteraient les larves de deuxième année de l'hôte dont les galeries sont alors envahies par un champignon du genre Amylostereum Boidin, lequel est probablement l'élément responsable de la localisation de I'hôte (Madden 1968). En outre, la larve de deuxième année de l'hôte a atteint la taille nécessaire aux importants besoins nutritionnels de la larve du parasitoïde atteignant rapidement $30 \mathrm{~mm}$ de longueur. Si ces hypothèses sont fondées, l'existence d'un cycle hôte-parasitoïde sur 2 ans est de nature à favoriser la coïncidence spatiotemporelle entre les deux espèces, M. atrata trouvant alors toujours, au cours de sa période de vol, son hôte dans l'état de développement adéquat. L'espèce serait alors typiquement idiobionte ( $\mathrm{Ha}-$ selbarth 1979) comme de nombreux autres ectoparasitoïdes.

\section{M. atrata dans la biocénose de l'orme}

L'orme américain est victime de la maladie hollandaise due au champignon Ceratocytsis ulmi (Buism.) qui est signalée dès 1930 en Ohio et en 1944 dans le sud du Québec. Le champignon pathogène introduit d'Europe aux EtatsUnis est véhiculé par Hylurgonipus rufipes Hbst. [Coleoptera: Scolytidae], américain, et Scolytus multistriatus Marsh. [Coleoptera: Scolytidae], introduit lui aussi d'Europe avant 1909, mais limité dans son extension vers le nord par les basses températures hivernales atteignant $-30^{\circ} \mathrm{C}$ (Strobel et Lanier 1981). Dans notre zone de prospection, les ormes hébergeant des $T$. columba dans le sud du Québec ne présentent pas de trace de Scolytidae, mais un système orme T. columba - Coléoptères vecteurs de champignons, écologiquement comparable à celui causant la maladie hollandaise, y est largement développé. $M$. atrata 


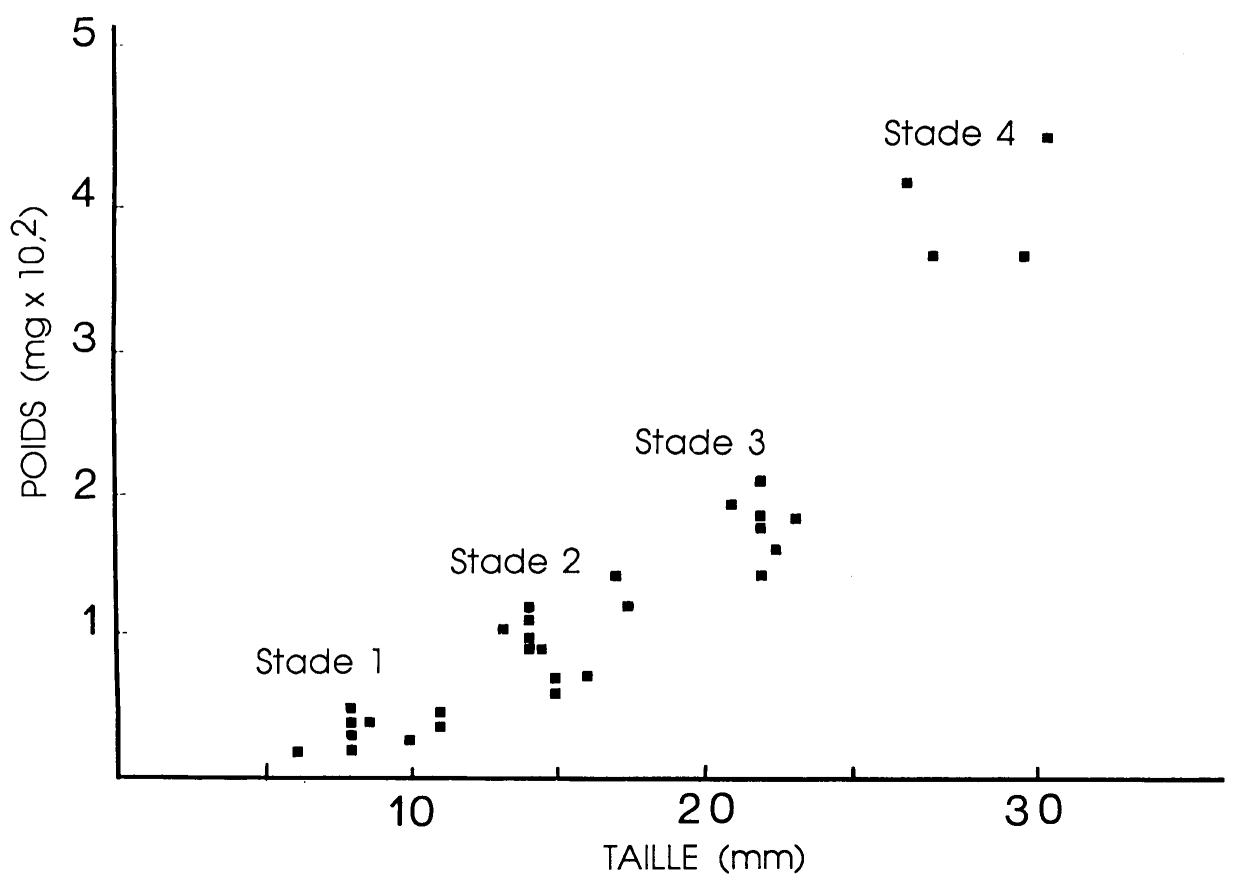

Figure 2. Mise en évidence des quatre stades larvaires de $M$. atrata en fonction du poids et de la taille des individus récoltés $(n=34)$ dans un volume de bois d'orme de $0,263 \mathrm{~m}^{3}$.

peut donc limiter les dégâts de cette maladie dont $T$. columba est le vecteur.

Les données peu nombreuses de la littérature sur le cycle de $T$. columba et totalement inexistantes sur celui de $M$. atrata autorisent, au vu de nos premières données, à supposer que les deux insectes associés par le parasitisme ayant une vaste répartition géographique holarctique néarctique accomplissent leur cycle de développement en 1 ou 2 ans, selon un gradient de températures moyennes annuelles décroissantes des ÉtatsUnis au Canada.

$T$. columba est un xylophage attaquant une large diversité d'arbres forestiers caducifoliés (Baker 1972; Muesebeck et al. 1951) lorsque ceux-ci sont morts, affaiblis mais aussi vivants (Baker 1972). Vecteur, lors de la ponte, de champignons qui envahissent les vaisseaux du bois à partir des galeries de ses larves, T. columba apparaît comme un ravageur forestier d'importance notable. II subit, par l'action de $M$. atrata, une limitation parasitaire dont l'efficacité est probablement liée à la détection de l'hôte par les champignons que sa présence induit. $M$. atrata apparaît d'autant plus intéressant comme agent de limitation des populations de Tremex qu'il peut grâce à la longueur de son ovipositeur atteindre les larves les plus profondément enfouies dans le bois. C'est à cette fin appliquée de protection des résineux que $M$. norto$n i$, originaire de la côte ouest-américaine, a été introduit avec succès en Australie pour contrôler Sirex noctilio F. [Hymenoptera : Siricidae] ravageur de Pinus radiata Don. (Talbot 1964) et Pinus ponderosa Dougl. (Nuttall 1973).

\section{REMERCIEMENTS}

Je remercie très vivement $M$. Guy Boivin, Ph.D. (Agriculture et Agroalimentaire Canada, Saint-Jean-sur-Richelieu, Québec) qui a largement facilité mes prospections de terrain et Mme M. Rault (Université de Rennes 1) qui a mis l'article en forme. 


\section{RÉFÉRENCES}

Abbott, C.E. 1934. How Megarhyssa deposits her eggs. J. N.Y. Entomol. Soc. 2 : 127-133.

Abbott, C.E. 1936. On the olfactory powers of Megarhyssa lunator (Hymenoptera: Ichneumonidae). Entomol. News 47 : 263-264.

Aubert, J.F. 1969. Les Ichneumonides ouestpaléarctiques et leurs hôtes. 1. Pimplinae, Xoridinae, Acaenitinae. Éditions Quatre Feuilles (avec le concours du C.N.R.S.), Alfortville, France. 302 p.

Baker, W.L. 1972. Eastern forest insects. USDA For. Serv. Washington, DC. 642 p.

Barlow, J. 1921. The mating habits of Megarhyssa (Hymenoptera : Ichneumonidae). Entomol. News 32 : 291.

Cameron, E.A. 1965. The Siricinae (Hymenoptera : Siricidae) and their parasites. Commonw. Inst. Biol. Control. Tech. Bull. 5 : 1-31.

Champlain, A.B. 1921. The curious mating habit of Megarhyssa atrata Fab. (Hymenoptera : Ichneumonidae). Entomol. News $32: 241$.

Cushman, 1926. Some types of parasitism among the Ichneumonidae. Proc. Entomol. Soc. Wash. 28 : 25-51.

Drooz, A.T. 1985. Insects of eastern forests, USDA For. Serv. Misc., Washington, DC. $426 \mathrm{p}$.

Fattig, P.W. 1949. Some observations on Megarhyssa. Entomol. News 60 : 69-71.

Fyles. 1916. Observations upon some of the predaceous and parasitic Hymenoptera. $46^{\text {th }}$ Annu. Rep. Entomol. Soc. Ont. : 52-60.

Gibbons, J.R. 1979. A model for sympatric speciation in Megarhyssa (Hymenoptera : Ichneumonidae) : competitive speciation. Am. Nat. $114: 719-741$.

Hardin, G. 1960. The competitive exclusion principle. Science (Washington DC) 131 : 1292-1297.

Harrington, W.H. 1887. The nuptials of Thalessa. Can. Entomol. 19 : 206-209.

Haselbarth, E. 1979. Zur Parasitierung der Puppen von Forleule (Panolis piniarius L.) und Heidelbeerspauner (Boarmia bistortata Loeze) in bayerischen Kiefern Wäldern. Z. Angew. Entomol. 87 : 311-322.
Heatwole, H. et D.M. Davis. 1965. Ecology of three sympatric species of parasitic insects of the genus Megarhyssa (Hymenoptera : Ichneumonidae). Ecology 46 : 140-150.

Heatwole, H., D.M. Davis et A.M. Wenner. 1962. The behaviour of Megarhyssa, a genus of parasitic Hymenopteran (Ichneumonidae : Ephialtinae). Z. Tierpsychol. 19 : 652-664.

Heatwole, H., D.M. Davis et A.M. Wenner. 1965. Detection of mates and host by parasitic insects in the genus Megarhyssa (Hymenoptera: Ichneumonidae). Am. Midl. Nat. $71: 374-381$.

Madden, 1968. Behavioural responses of parasites to the symbiotic fungus associated with Sirex noctilio F. Nature (London) 218 : 188-189.

Marchand, W. 1921. The egg laying habits of Megarhyssa. Science (Washington DC) 54 : 607-608.

Muesebeck, C.F.W., K.V. Krombein et H.K. Townes. 1951. Hymenoptera of America North of Mexico. Synoptic catalog. USDA, Agric., April, Washington, DC.

Nénon, J.P. 1993. Les Megarhyssa (Hymenoptera : Ichneumonidae) dans le sud du Québec : clé simplifiée de détermination et période de vol. Fabreries 18(2) : 49-58.

Nuttall, M.J. 1973. Pre-emergence fertilisation of Megarhyssa nortoni (Hymenoptera: Ichneumonidae). N.Z. Entomol. 5 : 112-117.

Riley, C.V. 1888. The habits of Thalessa and of Tremex. Insect Life $1: 168-179$.

Stilwell, M.A. 1966. The pigeon Tremex, Tremex columba (Hymenoptera : Siriciclae) in New-Brunswick. Can. Entomol. 99 : 685689

Strobel, G. et G. Lanier. 1981. La maladie hollandaise de l'orme. Pour la science, Octobre, 36 : 40-51.

Talbot, P.H.B. 1964. Taxonomy of the fungus associated with Sirex noctilio. Aust. J. Bot. $12: 46-52$.

Townes, H. et M. Townes. 1960. Ichneumon flies of America north of Mexico : 2. Subfamilies Ephialtinae, Xoridinae, Acaenitinae. Smithsonian Inst. Press, Washington, DC. $676 \mathrm{pp}$. 